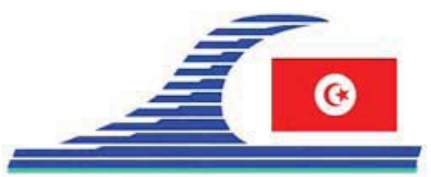

Conférence Méditerranéenne Côtière et Maritime EDITION 1, HAMMAMET, TUNISIE (2009)

Coastal and Maritime Mediterranean Conference

Disponible en ligne - http://www.paralia.fr-Available online

\title{
Étude du champ des vitesses interstitielles en zone de swash par Vélocimétrie Doppler Ultrasonore (VDU)
}

\section{Mustapha Kamel MIHOUBI ${ }^{1,3}$, Michel BELORGEY ${ }^{2}$, Ahmed KETTAB ${ }^{3}$}

1. École Nationale Supérieure d’Hydraulique (ENSH), BP 31, 09000 Blida, Algérie. mihkam@yahoo.fr;mk.mihoubi@ensh.dz

2. UMR-CNRS 6143, Laboratoire de morphodynamique continentale et côtière (M2C), université de Caen, 24 rue des Tilleuls, 14000 Caen cedex, France.

michel.bélorgey@orange.fr

3. Laboratoire de Recherches des Sciences de l'Eau (LRS-Eau), École Nationale Supérieure Polytechnique (ENP), avenue Hacène- Badi, BP 182, El Harrach, Algérie.

\section{Résumé :}

Le transport sédimentaire demeure toujours un phénomène complexe, les processus physiques qui lui sont associés n'ont été abordés jusqu'à maintenant qu'à partir d'analyses du champ de vitesses au sein d'une veine fluide. L'étude expérimentale de l'hydrodynamique des sédiments et l'analyse physique du processus d'échange à l'interface eau-sédiment en zone de swash (jet de rive) revêtent d'un intérêt capital pour la compréhension du processus de transport sédimentaire en milieu côtier, notamment par le contrôle et la prévision du trait de côte. L'objectif de cette étude a été d'exploiter la technique de Vélocimétrie Doppler ultrasonore (VDU) pour l'étude de l'évolution du champ des vitesses à l'interface eau-sédiment en zone swash pour différentes conditions de houles régulières incidentes en canal à houle.

Les vitesses sont mesurées pour différents situations du swash au sein du lit sédimentaire, et ce, dans la veine fluide de la langue de swash mettent en évidence : une répartition exponentielle des vitesses à l'intérieur du lit de sédiment; une différence de gradient de vitesse à l'interface eau-sédiment, entre la vitesse dans la lame d'eau de la langue de swash et l'écoulement interstitiel dans la nappe du swash. Les mesures obtenues ont permis de constater une évolution du profil des vitesses suivant les phases du swash, qui sont caractérisées par un déphasage entre l'écoulement libre de la langue de swash et l'écoulement interstitiel. Ces résultats suggèrent la nécessité d'une nouvelle approche d'analyse du transport sédimentaire qui doit tenir compte de la réalité des processus physiques qui régissent la phase d'interface eau-sédiment.

\section{Mots-clés :}

Vitesse interstitielle - VDU - Houle incidente - Porosité - Interface - Sédiment Swash 


\section{Introduction}

Le phénomène de transport sédimentaire est très complexe et les processus physiques qui lui sont associés sont encore mal connus.

En effet, faute d'une instrumentation appropriée, la modélisation de ce phénomène est encore basée sur deux types d'études indépendantes mais exploitées comme complémentaires, à savoir : (i) l'analyse du champ des vitesses dans l'écoulement libre, (ii) la mesure des vitesses interstitielles au sein de l'écoulement en milieu poreux uniforme à gradient hydraulique constant.

La grande majorité des études qui ont conduit à la modélisation du champ des vitesses dans l'écoulement libre permanent ont été réalisées pour un fond imperméable avec l'hypothèse de vitesse nulle au fond du lit. Les premières études relatives aux problèmes d'interface entre milieu poreux et veine fluide dans le cas d'un écoulement à surface libre sont très restreintes. BEAVERS \& JOESPH (1967), ont remarqué que dans le cas d'un écoulement confiné dans une conduite occupée par un bloc poreux, il peut y avoir un écoulement tangentiel non nul à la paroi. Il faut donc déterminer une nouvelle condition qui tient compte des caractéristiques d'écoulement en milieu poreux et à l'interface eau-sédiment.

Afin de mieux comprendre les processus physiques associés à ce phénomène, nous avons adapté la technique de vélocimétrie Doppler ultrasonore (VDU) à la mesure instantanée des vitesses interstitielles locales en zone de swash au sein d'un lit de sédiment d'une plage de type réflective pour les différentes conditions de houles régulières incidentes.

\section{Dispositif expérimental et technique des mesures}

Avant entamer cette étape expérimentale, nous avons réalisé des études de calibration et de validation des mesures de vitesses interstitielles ont réalisée sur un canal en écoulement à surface libre pour différents matériaux de lits perméables de : mousse alvéolaire, billes de verre et de sable moyen (MIHOUBI et al., 2008). Les expériences relatives à l'étude des effets de la houle incidente sur le profil des vitesses en zone de swash ont été menées dans un canal à houle régulière, d'une longueur de $18,0 \mathrm{~m}$ pour une section rectangulaire de 0,50 $\mathrm{m}$ de largeur et une hauteur des parois de 0,6 $\mathrm{m}$. Dans nos travaux nous avons simulé de nombreuses conditions de houles incidentes pour des périodes comprises entre $6,4 \mathrm{~s}<\mathrm{T}<1,90 \mathrm{~s}$. Le lit sédimentaire est constitué d'une plage qui s'étend sur une longueur de 2,0 m et une hauteur $0,3 \mathrm{~m}$. En fonction des conditions des houles incidentes et la position de formation du swash, nous avons prévu six colonnes de mesures (colonnes à sonde) ont été prévues dans la zone de formation du swash. 


\section{Analyse des résultats expérimentaux}

L'analyse du profil vertical des vitesses suivant les différentes conditions de houles incidentes ont permis de distinguer un déphasage entre l'écoulement libre de lame de swash et l'écoulement interstitiel dans le lit de sable (MIHOUBI et al.,2007). Sur la figure 1 on remarque bien le retard de l'écoulement interstitiel suivant la position verticale du swash. Les vitesses maximales enregistrées, durant la phase du jet de rive suivant les différentes conditions de houle incidentes (figure 2) ont permis de donner une forme de type exponentiel au profil vertical des vitesses dans le lit de sédiments et à l'interface de la en zone du swash (figure 3):

$\frac{\mathrm{u}}{\mathrm{u}_{\max }}=\mathrm{A}_{\mathrm{w}} \exp \left[\alpha\left(\frac{\mathrm{z}}{\mathrm{h}_{\mathrm{b}}}\right)\right]$

$A_{w}$ : paramètre caractéristique de l'hydrodynamique de l'écoulement en milieu poreux et de la vitesse à l'interface du milieu poreux-veine fluide.

$\alpha$ : coefficient qui caractérise la nature du champ des vitesses dans le lit sédimentaire. Il ne dépend que de la porosité du milieu et des conditions de la houle incidente.

On pose : $\mathrm{U}=\frac{\mathrm{u}}{\mathrm{u}_{\max }}$ et $\mathrm{Z}=\frac{\mathrm{Z}}{\mathrm{h}_{\mathrm{b}}} \quad$ avec, $\quad \mathrm{Z}<0$

A partir du tracé de la fonction $\ln (\mathrm{U})=\mathrm{f}(\mathrm{Z})$, on déduit directement la valeur du paramètre $\alpha$ caractérisant l'écoulement interstitiel dans le lit sédimentaire. Les expériences ont montré que la valeur de " $\alpha$ " dépend des caractéristiques de l'écoulement généré par de la houle en zone de swash et des paramètres physiques du milieu poreux tels que la porosité du milieu, la conductivité hydraulique et le diamètre des pores ou interstices du lit de sédiments.

\section{Conclusions}

L'analyse du champ des vitesses maximales dans la phase de jet de rive met en évidence un profil vertical des vitesses de forme exponentielle qui dépend non seulement des caractéristiques du lit : porosité, conductivité hydraulique, régime d'écoulement dans les pores et interstices des sédiments mais il est lié aussi aux conditions de la houle incidente, notamment de sa période. Dans ce contexte, nos essais ont mis en évidence des relations non linéaires entre le paramètre " $\alpha$ ", qui caractérise le champ des vitesses dans le lit sédimentaire, et la période de la houle incidente ainsi que pour le régime local d'écoulement dans le lit sédimentaire. Les résultats obtenus par les mesures des vitesses par VDU suivant l'écoulement sur un lit perméable montrent que les modèles classiques de détermination de la contrainte de frottement $\tau_{\mathrm{p}}$ à la paroi ne sont pas bien adaptés, du fait, qu'ils sont basés principalement sur les caractéristiques de l'écoulement libre sans tenir compte de la nature du lit de sédiments et de ses caractéristiques en particulier la porosité et le régime d'écoulement local interstitiel en fonction du nombre de Reynolds des pores. 


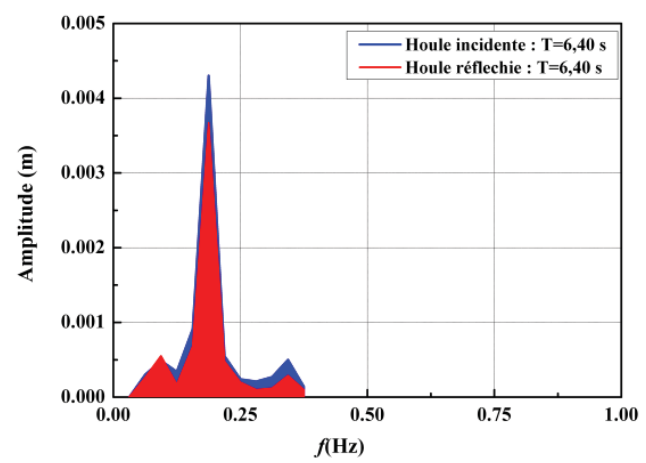

Figure 1. Spectre de la houle incidente : $T=6,4 \mathrm{~s} ; H=0,45 \mathrm{~cm}, d=0,285 \mathrm{~m}$
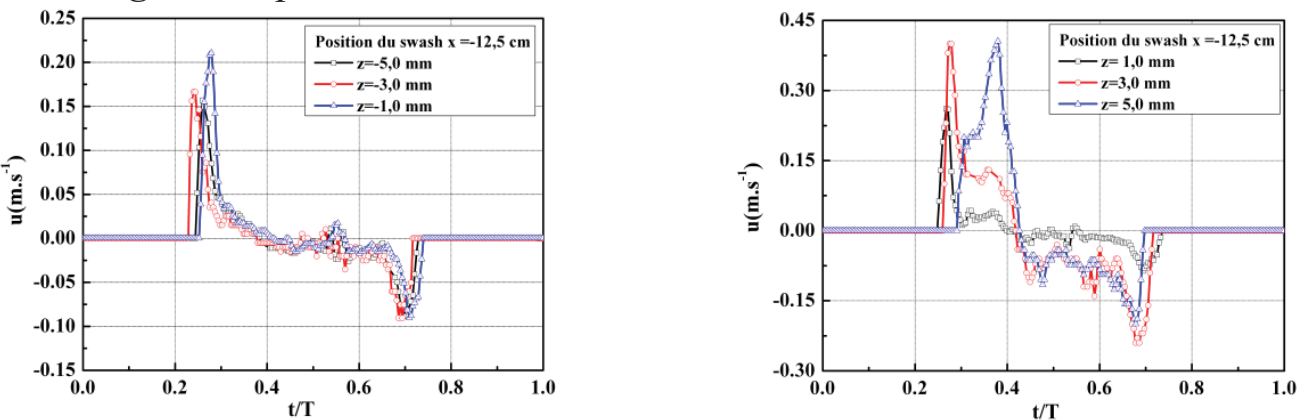

Figure 2. Evolution du profil des vitesses instantanées du swash à différentes hauteurs $z$ : pour une houle incidente : $T=6,4 \mathrm{~s}, d / L=0,017$.
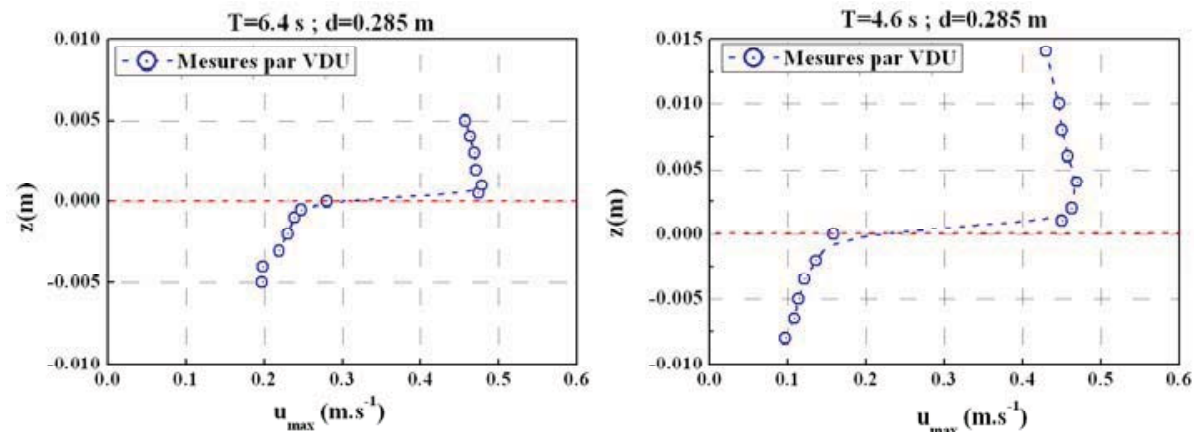

Figure 3. Profils des vitesses maximales en phase de jet de rive (uprush),

(Figure de gauche : $T=6,4 \mathrm{~s} ; d=0,285 \mathrm{~m}$. Figure de droite : b) $T=4,6 \mathrm{~s} ; d=0,285 \mathrm{~m}$ ).

\section{Références bibliographiques}

BEAVERS G.S., JOSEPH D. (1967). Boundary conditions at a naturally permeable wall. Journal of Fluid Mechanics, 30, pp 197-207.

MIHOUBI M.K, BELORGEY M., KETTAB A, LEVACHER D. (2007). Experimental approach study of interaction water-sediments in the swash zone by ultrasonic Doppler velocimetry, proceeding of $15^{\text {th }}$ IAHR, symposium on River, costal and estuarine morhodynamics (RCEM'07), University of Twente, pp 345-350.

MIHOUBI M.K., BELORGEY M., KETTAB A. (2008). Analysis, in a free surface steady flow, of the interstitial velocity field inside a sedimentary bed. C. R. Geoscience 340, pp 858-864. 